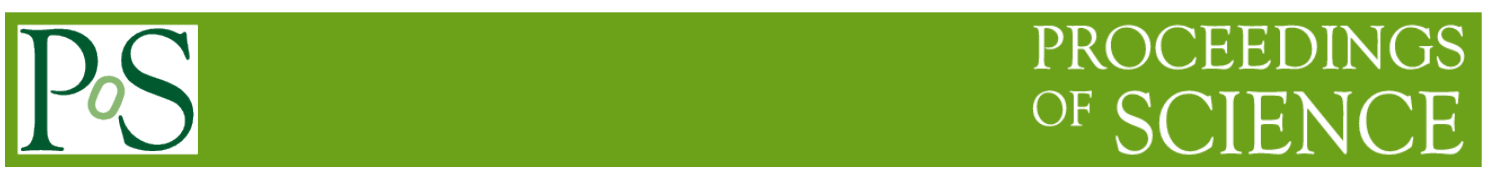

\title{
The new front end and DAQ of the ICARUS detector
}

\section{Guang Meng*}

INFN PADOVA, Via F. Marzolo 8, 35131 Padova, Italy

E-mail: guang.meng@pd. infn. it

Icarus is the largest imaging LAr TPC ever operated. During LNGS run on the CNGS neutrino beam, from 2010 to 2013, Icarus produced some thousands neutrino events of unprecedented quality. This was possible thanks to precision and stability of mechanics, liquid argon purity and high $\mathrm{S} / \mathrm{N}$ of electronics. In this poster the last issue (electronics and DAQ) will be presented in detail. Presently Icarus T600, in view of its operation at FNAL on the SBN neutrino beam, is undergoing major overhauling that implies cathode mechanics improvement, additional PMTs installation and a new electronics front-end and DAQ. This new electronics is integrated onto the flange proprietary design, with a new front-end that improves $\mathrm{S} / \mathrm{N}$ and induction signals treatment. Also this issue will be presented in detail together with data recently recorder at CERN in the Icarino, 50-litre, LAr facility.

The European Physical Society Conference on High Energy Physics

5-12 July, 2017

Venice

* on behalf of Icarus collaboration 


\section{Introduction}

The Icarus electronics, used in the LNGS run, performed efficiently with an extremely good $\mathrm{S} / \mathrm{N}$ that allowed for collecting some thousands neutrino and cosmic events with unprecedented quality. The overhauling of the T600 gave the opportunity, among other improvements, to design new electronics, that integrates the electronics modules itself onto the signal flanges with dramatic volume reduction. At the same time a new front-end amplifier more compact and with different signal shaping was implemented. The poster presents the new electronics together with tests results obtained on the 50-litre liquid argon (LAr) TPC facility in CERN.

\section{General architecture}

The architecture adopted is the same as the one in the original design (1998), but using more performing modern components.

For what concerns the front-end, multiple $\mathrm{jFets}$ in parallel at input of each amplifier were adopted to increase the total $g_{m}$. Smaller package ASIC, custom design, was chosen for compactness.

The input stage is followed by a shaper (classic zero-pole cancellation filter) and baseline restorer with peaking time of $1.6 \mu \mathrm{s}$ in response of a delta-like input current. The basic scheme is given in figure 1.

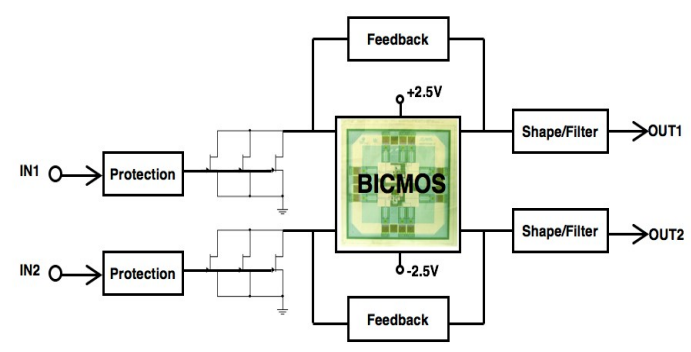

Figure 1. Block diagram of the dual channel front-end amplifier with the ASIC and external networks for feedback and filtering.

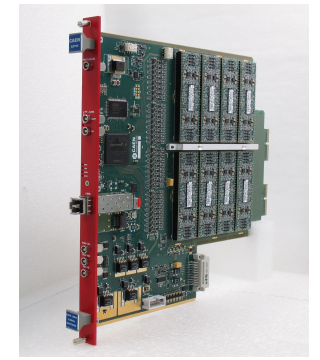

Figure 2. Picture of the board A2795. The front part contains the digital section while in back part front-end amplifiers (64 channels) are visible, grouped in sets of 8 channels per piggy-back modules

Same shaping time has been chosen both for induction and collection signals. This feature allows for better handling of induction signals, with a significant increase of $\mathrm{S} / \mathrm{N}$. Preserving the bipolar shape of the induction signals allows for easy treatment and energy reconstruction also for dense showers.

Each amplifier is followed by a serial 12bit ADC, with sampling frequency of $2.5 \mathrm{MHz}$. This choice allows for same sampling rate as in the old system (400ns per channel), avoiding cumbersome use of multiplexers. The data stream of each channels is fed into a highperformance FPGA, that performs data compression, buffering, and transmission via optical link to DAQ. 
Analogue and digital electronics for 64 channels, are housed in a single board, Figure 2, that fits into the external connectors of the flange. The board A2795 was designed and implemented in close cooperation with CAEN S.p.A.

On each flange a custom crate hosts the nine boards ( 576 channels).

\section{Tests with cosmic rays and results}

Tests were performed in the 50-litre LAr TPC at CERN, with drift field of $500 \mathrm{~V} / \mathrm{cm}$ on a drift length of $52 \mathrm{~cm}$.

The wire chamber is approximately $32 \mathrm{~cm} \times 32 \mathrm{~cm}$ wide and has three wire planes with $4 \mathrm{~mm}$ separation, while the wire pitch on each plane is $2.54 \mathrm{~mm}$. The first plane, grid, facing the drift volume, is not read, the intermediate plane, induction, has 128 wires parallel to the ones of the grid. The last plane, collection, has the 128 wires orthogonal. The grid plane is biased at $-300 \mathrm{~V}$, the induction at $0 \mathrm{~V}$, and the collection at $+300 \mathrm{~V}$ to guarantee grid and induction transparency.

The setup is shown in figure 3 where the 9 A2795 are inserted in the special crate mounted on the feed through flange.
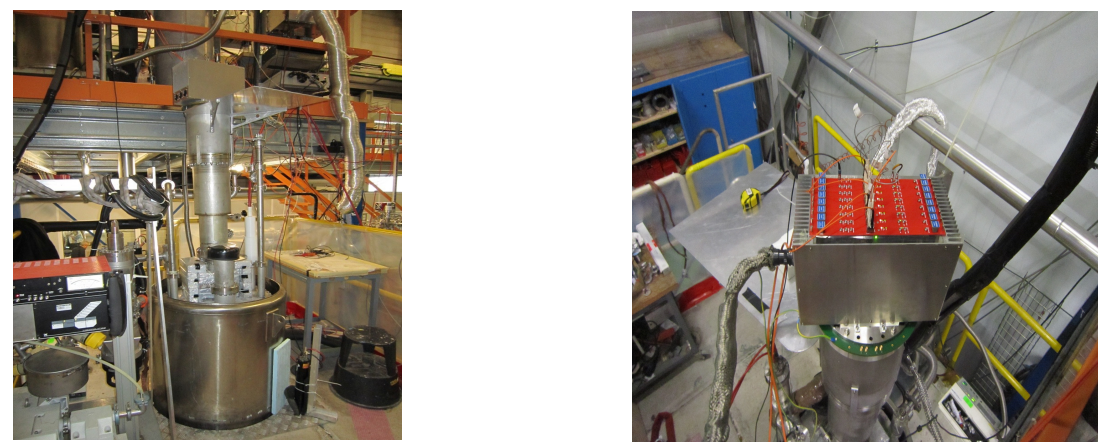

Figure 3. In the left the full test facility (dewar and electronics) is shown, while in the right the detail of the crate housing 9 boards, mounted on top of the signal flange, is visible.

A single m.i.p. track in collection and induction is shown with its signal profiles in Figure 4. Equivalent Noise Charge (enc) is $1000 \mathrm{el}$. No filter applied to any data.
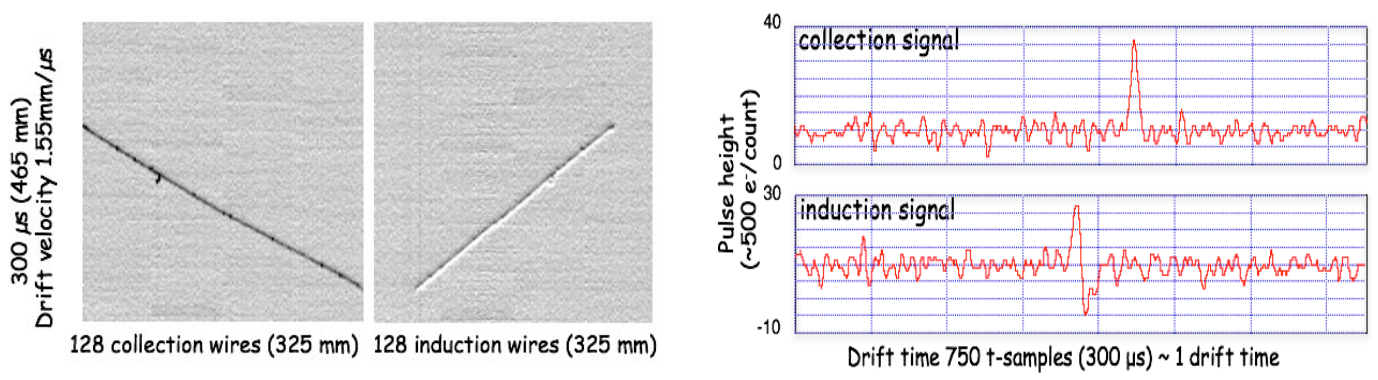

Figure 4. A single m.i.p. track in collection and induction. Bipolar structure of the signal is preserved both in image and signal profile.

A shower event is given in Figure 5. In the induction image the bipolar structure is preserved and no under-shoot is present, due to the new shaping of the amplifier. 

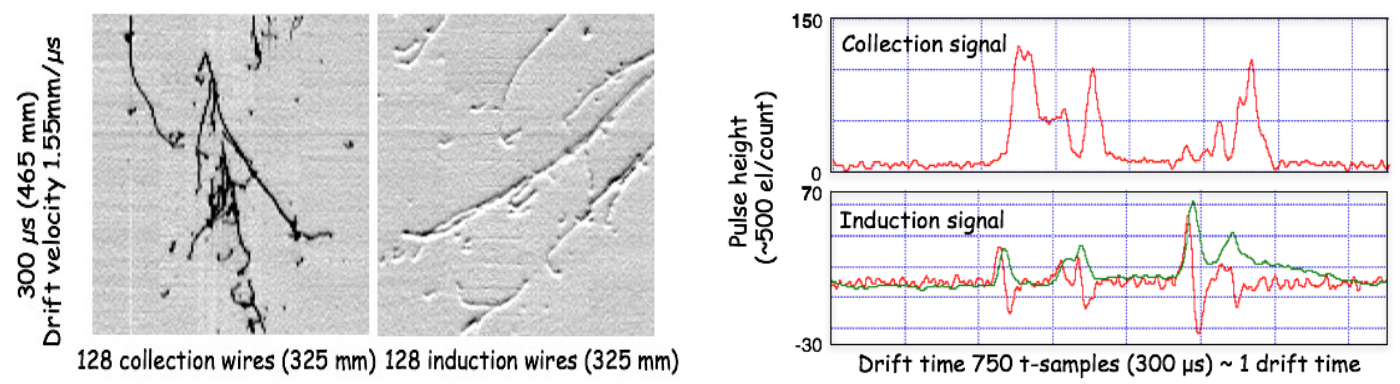

Figure 5. A shower event in collection and induction. Bipolar structure of the signal is also in this case preserved both in image and signal profile.

Muon tracks were used to calibrate the electronic chain against minimum ionizing particles $(2.1 \mathrm{MeV} / \mathrm{cm}$ in LAr).

The rms noise is 1.1 counts at $40 \mathrm{pF}$ detector capacitance, 2 counts at $143 \mathrm{pF}$, and 2.4 counts at $194 \mathrm{pF}$.

\section{Conclusions}

The new front end and DAQ for the ICARUS detector have been designed, successfully tested and the electronics boards are now in production by CAEN that was awarded for the supply. The new design is a significant step forward compared with the old electronics both in terms of S/N and energy resolution. Moreover, after the agreement between INFN and CAEN, the digital board A2795 is now an off the shelf product in CAEN catalog.

The board can be the standard solution for medium-large volume LAr TPC with limited ( 4 meter) internal cable length.

The A2795 is a viable solution also in case of LAr TPC with cold front-end. In this latter case, an ad hoc receiver will replace on board amplifier modules.

\section{Acknowledgments}

Icarus collaboration wants to thank A. Mati and A. Romboli from CAEN for their effective support in the development and design of the hardware and firmware of the digital part, and $\mathrm{M}$. Nicoletto and his coworkers of Laboratorio di Elettronica of INFN/Padova for the continuous and fundamental contribution in the development of analogue part, low noise power supply, and testing of the full system. 